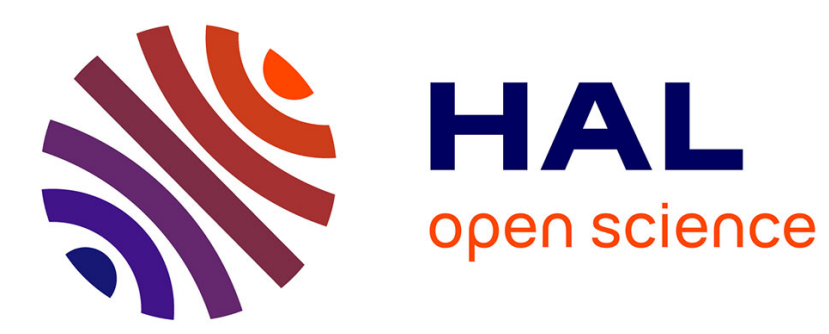

\title{
Information Centric Fog Network for Incentivized Collaborative Caching in Internet of Everything
}

\author{
Junaid A. Khan, Cedric Westphal, Yacine Ghamri-Doudane
}

\section{To cite this version:}

Junaid A. Khan, Cedric Westphal, Yacine Ghamri-Doudane. Information Centric Fog Network for Incentivized Collaborative Caching in Internet of Everything. IEEE Communications Magazine, 2019, 57 (7), pp.27-33. 10.1109/MCOM.2019.1800764 . hal-02148782

\section{HAL Id: hal-02148782 \\ https://hal.science/hal-02148782}

Submitted on 5 Jun 2019

HAL is a multi-disciplinary open access archive for the deposit and dissemination of scientific research documents, whether they are published or not. The documents may come from teaching and research institutions in France or abroad, or from public or private research centers.
L'archive ouverte pluridisciplinaire HAL, est destinée au dépôt et à la diffusion de documents scientifiques de niveau recherche, publiés ou non, émanant des établissements d'enseignement et de recherche français ou étrangers, des laboratoires publics ou privés. 


\title{
Information Centric Fog Network for Incentivized Collaborative Caching in Internet of Everything
}

\author{
Junaid Ahmed Khan*, Cedric Westphal ${ }^{\dagger}$ and Yacine Ghamri-Doudane ${ }^{\ddagger}$ \\ ${ }^{*}$ Center for Urban Science and Progress (CUSP), New York University, NY, USA \\ ${ }^{\dagger}$ Huawei Technology, Santa Clara, CA, USA \\ ${ }^{\ddagger}$ L3i Lab, University of La Rochelle, France
}

\begin{abstract}
Current mobile network infrastructure has a hard time keeping up with the constant content demand by an increasing number of smart devices, both in terms of bandwidth and cost. At the same time, the advent of devices with relatively high resources (computing, communications, caching) allows to offload computation, control, cache and communication near the users as in Edge or Fog Networking. If we consider the caching resource, the basic challenges are: (i) How and who can form fogs for local content caching? and (ii) How to interact with the fog for efficient content caching and retrieval? To address these, we leverage Information Centric Networking (ICN) to propose IS-Fog, a social-aware fog network where the content is the first class citizen. We first classify a device's eligibility to provide content using a novel content-based centrality. At the same time, we incentivize users to self-organize and share their device's resources and cache in the fog. We then propose a social-aware content caching and a distribution scheme to model fog interactions allowing nodes to collaboratively cache content locally. We evaluate IS-Fog using realistic mobility traces of 2,986 nodes. Results shows that IS-Fog is a scalable and efficient content caching and distribution approach compared to existing schemes.
\end{abstract}

Index Terms-Information Centric Networking, Fog Networking, Content Caching, Internet of Everything (IoE)

\section{INTRODUCTION}

Future smart city applications are expected to generate and consume a large amount of data with intelligent devices related to connected transportation (smart vehicles), buildings (smart home), industry and manufacturing, energy sector (smart grid) , e-health (wearables), surveillance, agriculture (sensors and actuators) and mobility-related applications (smart phones). The Internet of Everything (IoE) paradigm will allow every thing to connect to the Internet [1], [2]. At the same time, the soaring number of such connected things pose an alarming situation: Cisco predicts that by 2020 , around 50 billion devices will be connected to the Internet [3].

It is challenging for current mobile network infrastructure to deal with such massive content delivery to users. For instance, different users requesting the same recently aired episode of a popular TV show on their mobile devices in an urban environment would result in lots of redundant requests in close proximity. Cloud based solutions help a lot. However, they will be strained under current demand growth rates. Today, augmented/virtual reality or vehicular automation applications requires quick response, under variable network connectivity and availability, while clouds incur long setup times, are leased for long time and are oblivious to network connectivity.
Fog networking is a promising network architecture for devices at the edge (near user) to perform local decentralized resource pooling (computing, caching and communication) as an ad-hoc network, rather than using the infrastructure network [4], [5]. It addresses the end users requirements for real time processing of content "Now and Here" while supporting rapid innovation and affordable scaling for increasing number of multimedia rich Augmented/Virtual Reality (AR/VR) based applications in the upcoming $5 \mathrm{G}$ networks. We believe spatio-temporally co-located user devices with relatively high resource such as routers, small cells, and even vehicles can mutually orchestrate resources and act as surrogates for the infrastructure in the 5G networking architecture to compute, cache and communicate locally near the end users. Users in a neighborhood can enable their set-top boxes/ wireless Access Points (APs) or vehicles On-Board Units (OBUs) to mutually cache and share that popular TV show episode, thus limiting the back-haul connections to the service/content provider from other nearby users.

Two basic questions needs to be addressed: (i) How to identify and provide incentives to suitable candidate users with devices, capable to compose, deploy and manage a distributed fog network? and (ii) How devices in a fog interact and collaborate to efficiently cache and retrieve content?

To address these questions, we present an Informationcentric Social-aware Fog (IS-Fog) networking where Information Centric Networking [6], [7] with its in-network caching [8] and content provider-consumer decoupling along "anycast" routing support can be combined with Fog Networking. Additionally, we advocate a social aware approach where we re-define centrality, a measure used in network sciences to identify important nodes in a graph. Identification of influential users is required in social networks (and other graphs [9],[10]) for disseminating information.

Therefore, we first identify eligible candidate devices using a new content-based centrality scheme which classify a device based on its ability to provide content to users, instead of its topological connectivity. Such devices are viable candidates to provide their caching, computing and communication resources to facilitate content delivery to nearby devices. Second, we target the fog formation where we exploit gametheoretic concepts to motivate co-located devices to form a coalition and collaboratively cache maximum content locally in a distributed fog. Finally, we propose a socially aware content caching and distribution scheme modeling intra- and 


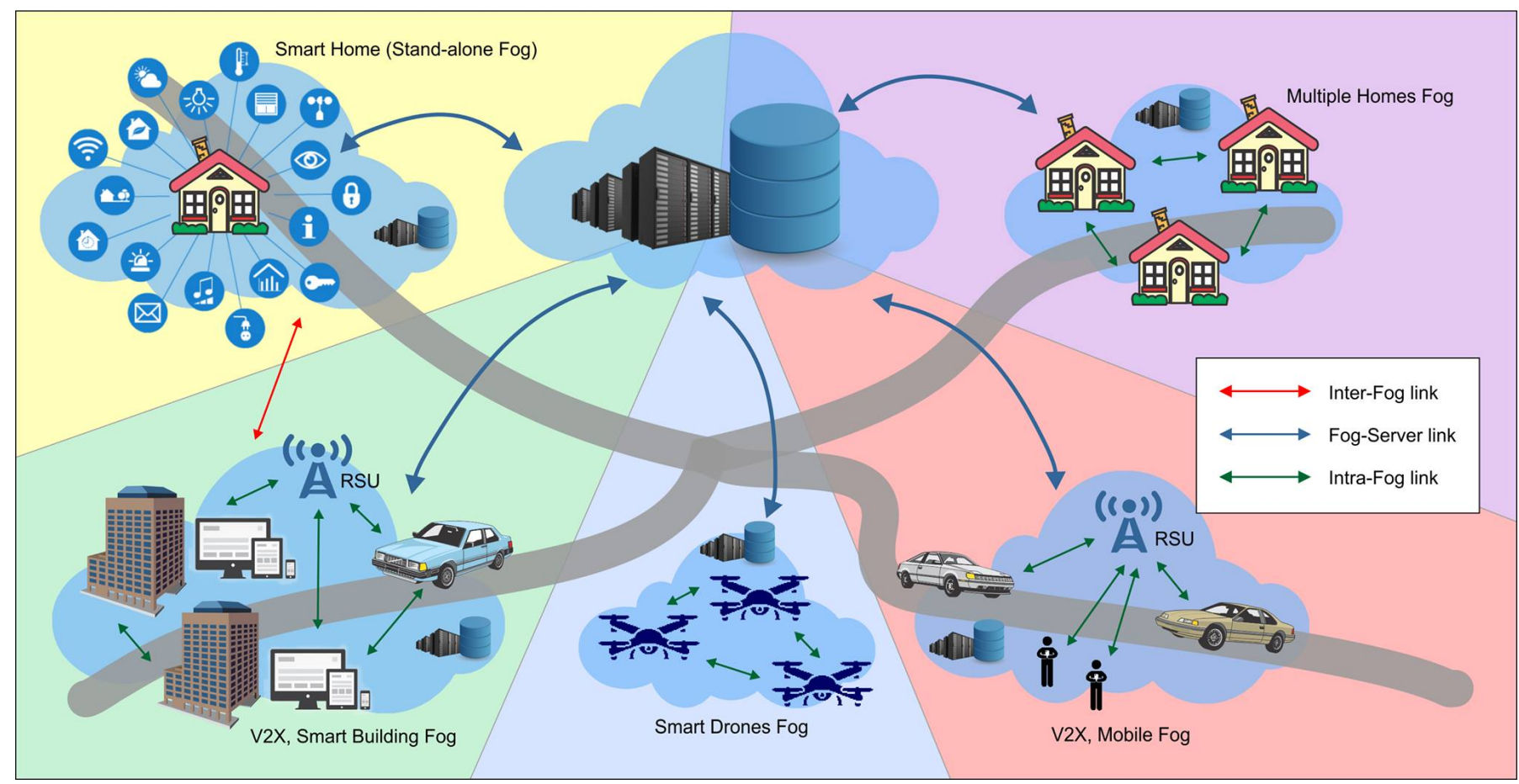

Figure 1: IS-Fog System Overview

inter- fog interactions where ICN enabled "any-cast" routing toward high centrality devices is used for cooperative content retrieval.

The goal is to provide a low cost social aware distributed caching, computing and communication infrastructure at the edge that will complement the existing infrastructure and will offload network traffic, extend coverage, improve performance, and reduce the dependence on cloud-based solutions.

The next section presents the system overview of IS-Fog. In Section III, we discuss the device identification phase where we redefine centrality scheme to classify a node. Section IV describes the incentivization phase for fog formation. Section $\mathrm{V}$ targets the collaboration phase where we describe the fog interactions in order to achieve efficient content caching and retrieval. An example discussing the joint identification, incentivization and collaboration is provided in Section VI. The performance evaluation and the results are discussed in Section VII. Section VIII highlights some open research issues towards Information-centric Social-aware Fog Networking. Finally, Section IX concludes the article.

\section{IS-Fog: The BIg Picture}

We present IS-Fog as a framework that brings computing, control, caching and communication near the edge devices (locally) to complement the infrastructure network. We leverage fog networking as an architecture that uses nearby user devices to perform collaborative sharing of such resources. Figure 1 shows a system overview of different fog networks targeting different IoE applications in a smart city. Fogs can comprise of different devices, i.e. connected smart vehicle automation, network of smart drones, smart home automation [11], even multiple houses and vehicles can collaborate to mutually form a fog in a neighborhood.

IS-Fog aims to use the ICN name-data networking paradigm with any-cast routing for content caching and distribution in order to maximize content availability at the network edge. We suggest using ICN as a better suited architecture with its data centric approach along the support for in-network caching towards an integrated solution. However, the proposed collaborative caching is independent of the underlying layer 3 architecture and therefore, the current IPv4 based Internet infrastructure can still be used to implement our solution.

The first step is to find the best possible candidates who can be motivated to be part of distributed fogs. Then, such candidate devices can self-organize for efficient social-aware content caching and distribution in the network. IS-Fog can scale from a standalone smart home with different connected devices (no-mobility, indoor) to city-wide fleet of thousands of interacting autonomous vehicles (highly mobile, outdoor) though we describe below concisely two use cases where ISFog is applicable:

Smart Home/Building Fog: An interesting fog use case is a smart home/building comprised of different connected devices/sensors where their data should to be collected, either periodically or in an event-based manner, then pre-processing techniques need to be applied to the collected data which requires sufficient computing powers. Similarly, useful preprocessed data needs to be stored and communicated locally using low cost resources for efficient access as its intended utilization lies locally. However, such data collection is challenging due to the heterogeneous resources over different devices, including constrained devices with limited resources. Similarly, subscribers requesting video streaming services 
from providers such as Netflix can be an example of largescale traffic in a small building.

Thus, there is a need to find devices with relatively high computing, caching and communication capabilities to collaborate and offer resources to perform such actions. Moreover, we also need a controller or facilitator within the fog to decide how and when to perform efficient content caching and distribution based upon the user(s) interest. Such controller is also required to deal with devices in the fog belonging to different manufacturers/owners, thus requiring efficient collaboration policies while ensuring fairness.

Vehicular Fog: Another use case targets an outdoor urban environment with highly mobile devices such as smart vehicles and drones. For example, smart vehicles today equipped with different heterogeneous sensor platforms can collect, preprocess, store and share data from urban streets due to their relatively high computing, caching, and communication capability [12]. However, it is challenging to collect data generated from, and consumed by, a large number of vehicles. To achieve a scalable city-wide data collection and distribution, there is a need to find suitable vehicles with high inherent caching, computing and communications capabilities to collaborate.

The aforementioned use cases require the availability of relevant content "Now and Here" for the users regardless of the source IP address. Therefore, IS-Fog comprises a publishsubscribe ICN model allowing any user device (anything with wireless communication unit supporting 5G, LTE or Wifi) to subscribe to one (or more) of three roles: information provider, facilitator and consumer.

We consider an application where different entities can subscribe to provide their resources, such as caches in this case to the service provider and in return, be rewarded (for instance, with coupons or service discounts). Therefore, different owners can subscribe for smart home services and collaboratively share their resources, irrespective of their appliance vendors.

The three distinct roles are defined since certain user devices are capable to be subscribed only as consumers or providers depending on the available resources, therefore not participating to facilitate other subscribers in the network, although devices with multiple role can exist in IS-Fog.

\section{IDENTIFICATION: REDEFINING CENTRALITY}

It is non trivial to find the devices available at the right time and place to form fog in order to facilitate users in the network. We believe that, among all the subscribed user devices, only a set of appropriate devices with sufficient resources can be considered important. However, the question is, how to identify and select devices for content caching, distribution and retrieval in a fog? To address this, we define a scoring/reputation system where high centrality user devices are considered the best candidates based on their ability to provide content. The ad-hoc network connectivity between devices in a fog can be modeled by a graph and therefore the well known centrality concept from network sciences can be used to identify important nodes in a graph.

We allow high centrality devices to act as a coordinator to create or merge/leave distributed fogs by collaborating with

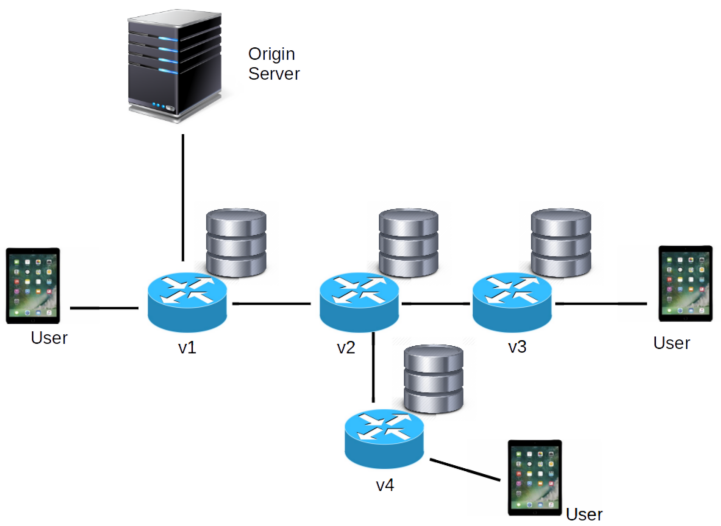

Figure 2: Content-based Centrality Example

nearby devices while at the same time considering the user interests. Incentives can be provided to motivate them [13].

A smart home can choose set of relatively powerful devices while a set of smart vehicles can self-organize between them to form distributed fog for efficient resource pooling in a neighborhood.

We define a novel content-based centrality measure as the sum of the ratio of the number of shortest paths in terms of number of hops from all users to all content that passes through the node to the total number of shortest paths between all the (user,content) pairs weighted by the popularity of the content. Figure 2 shows an illustration to explain the concept. Here the node $v_{1}$ is on the path from the users to the content in the server and requests will go through $v_{1}$ and therefore $v_{1}$ will have very high centrality. It is easy to see that the usual topological definition of centrality should therefore be modified as it varies on the type of content and where this content is cached.

Content that is popular will generate more traffic, therefore the nodes that offer paths for this content should also have a higher content-based centrality.

Once the device computes its centrality, it can consider itself to be part of a distributed fog by either (i) creating a new fog in case there is no existing fog in the vicinity or (ii) merge into an existing nearby fog created by high centrality devices. Nearby fogs may be formed by different owners. We also assuming a monetary reward is paid to the owners of the devices forming such fog. The users would prefer to merge into a fog with larger rewards formed by high centrality devices in order to gain a bigger payoff (say, proportional to the overall resources offered). However, the reward is based on the overall content delivered by the fog to which it belongs. We allow the identified high centrality user devices to cooperate in order to better manage and control distributed fogs due to their relatively high capabilities. To ensure fairness and provide incentives to caching nodes, game-theory based solution can be used. One such solution is discussed in the next section.

\section{InCEntivizATION: Fog Formation}

We incentivize users to collaborate and offer their device's storage buffer to cache content locally where the process is 
referred to as fog formation for distributed caching. To manage this, we borrow the game-theoretic concept of coalition formation where the goal is to offload a maximum amount of content from the service provider locally at the nodes subscribed to cache at different locations and times.

\section{A. Coalition formation}

Smart devices with caching capability can participate to form a coalition in a smart home/building . An optimization problem with the objective to cache a maximum amount of content locally near the end users can be modeled, while maximize the joint utility of nodes caching in the coalition for the users in the building/neighborhood. However, the constraints can differ between highly mobile devices such as connected vehicles, and stationary caching devices such as routers, small cells etc. However, common constrains such as cost requires to compensate for the resources spent such as the consumed energy and storage space of the participating devices. It can be in the form of a monetary reward, concurrency, coupons/fidelity points on subscription or possible gift cards with a common goal of compensating users for their energy and storage. Another important constraint is the social interest and as the incentiviation takes into account owners/users with similar social interests based on their centrality score in the fog. Neighnors commonly interested in a particular TV series can collaboratively cache and share a recently aired episode. The spatio-temporal coalitions formed, thus reflect such social similarity among participants. Therefore, the reward offered by the content provider should be such that users caching content at their devices are themselves interested in the content and are not required to cache uninteresting content.

\section{B. Solution concept: The grand coalition formation}

The solution of the coalition game targets to form the set of fogs where we associate to each coalition $s$ a value $U_{s}(l, \bar{t})$ as the total reward available to players (caching nodes) in the coalition $s$ at the location $l$ and during time-slot $\bar{t}$ in an urban environment. The coalition reward can be computed as the total amount of content delivered to the end users by the caching nodes in the coalition. To increase the coalition utility, we should allow high centrality nodes to form and manage the fogs and cache a diverse content set in the coalition. Each individual node subscribed to cache prefers to merge to a nearby coalition with larger utility in order to increase its reward. Given two coalitions $s_{1}$ and $s_{2}$ in the node $v$ vicinity, $s_{1} \succ s_{2}$ indicates that node $v$ prefers to merge with $s_{1}$ over $s_{2}$ assuming $s_{1}$ is initialized by a high centrality node along nodes caching diverse content set. By joining $s_{1}$, it increases the coalition utility $U_{s_{1}}(l, t)$ since together with $s_{1}$, it can cache content which will result in $s_{1}$ delivering more amount and diverse set of content to the nearby users, and consequently increasing the node reward. Therefore, the more diverse set of content is cached and subsequently delivered to the users by the nodes in coalition, the more the collective reward the nodes in the coalition receive.

The stable solution concept for the coalition game, namely the core can be achieved where each node subscribed for caching is motivated to participate towards forming the grand coalition of all nodes at a given location and time. It subsequently receives a share from the coalition reward proportional to the amount of content delivered altogether by the respective coalition. Under such conditions, a maximum amount of content will be efficiently cached at the nodes in coalition as stated in the above objective function while at the same time increasing diversity in the set of content available locally for the end users.

\section{COllaboration: InTER/InTRA Fog InTERACTIONS}

The focus of this section is the interactions between distributed fogs formed by the high centrality devices identified in the previous section to facilitate social aware content caching and distribution. The interactions and collaborations are classified into two categories, intra-fog and and inter-fog.

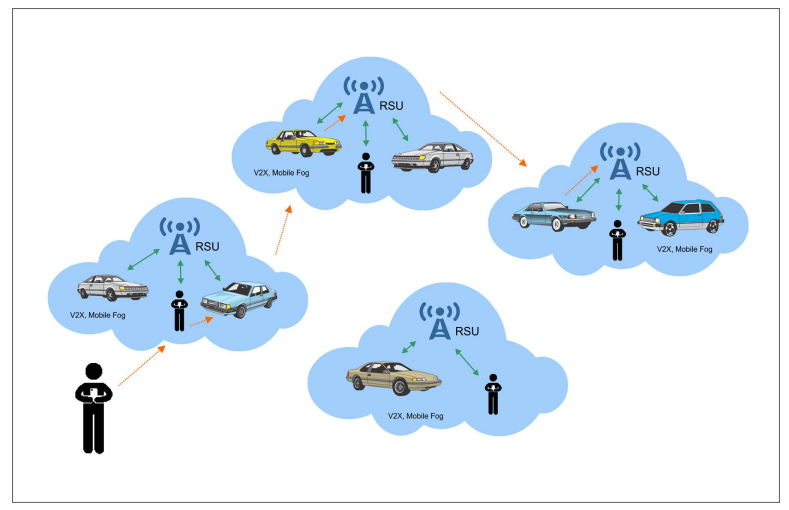

Figure 3: Facilitator Discovery Process

\section{A. Intra-fog collaboration}

It is the collaboration between different information facilitator devices in the fog to decide resource management for efficient content caching and distribution. High centrality user devices in each fog can collaborate with other nearby nodes to cache content. Content set is offloaded at set of user devices , while respecting each device's buffer threshold. The content placement process is initialized by the user with the highest centrality device in a neighborhood. It can announce itself as the responsible node for the neighborhood after a contention and start caching the most popular content while respecting its buffer constraints, i.e. the cached content should not exceed the available buffer space. The remaining content are cached in a similar fashion with their decreasing popularity at nearby devices with decreasing centrality where a maximum content is cached locally with their decreasing popularity order. Finally, the set of content and devices in the fog are updated with the cached content. The coalitions described in the previous section can be applied where a device not merging with an existing nearby coalition formed by high centrality devices and greedily caching most popular content will in turn receive less payoff as the same popular content is already provided to the users by the high utility coalition comprising high centrality (content providing) devices. 


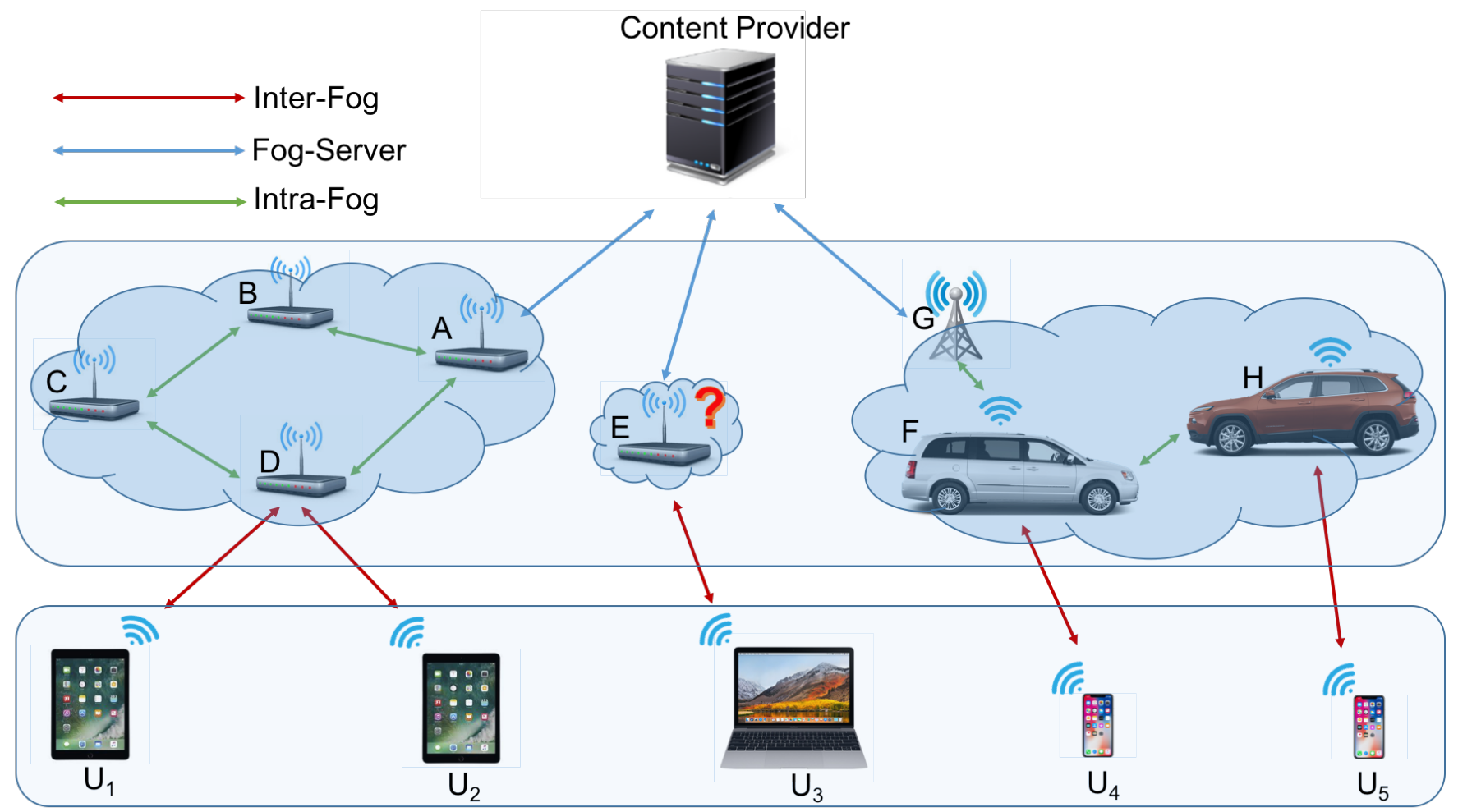

Figure 4: Example: IS-Fog Summarized

\section{B. Inter-fog collaboration}

Three types of interactions are possible in IS-Fog, (i) FogConsumer for user interests to be forwarded to near-by devices in the fog, (ii) Fog-Provider interaction for content retrieval from information providers and (iii) Fog-Infrastructure interplay where infrastructure assisted fog networking enables to use infrastructure as a last resort resource in case content not available locally.

A consumer can forward its name-based interests using ICN inherent"any-cast" routing towards high centrality information providers. The idea is to let the user "pull" content of interest cached at important information facilitators at a local scope and reduce network overhead. We use the content-based centrality P-CBC to discover nearby information facilitators. A user interest can also specify a deadline to indicate the maximum threshold time to facilitate the requested content. Thus, in case the interest cannot be satisfied by a local facilitator at nearby fog by a threshold, the user interests can be forwarded to infrastructure network to avoid high delays in content retrieval. The benefit of any-cast routing here is to enable multiple nearby facilitators to respond to the user interest in order to improve the content retrieval efficiency.

Figure 3 shows the fog interactions for the social aware content distribution and retrieval where a consumer-generated interest for content is forwarded to a high centrality facilitator in the fog. A facilitator discovery process initializes and continues to search for the content at each intermediate relay fog by constantly discovering the next high centrality device in each intermediate relay fog since such a device have the ability to provide content. If it is unable to find the content in the cache of all the member facilitators, it performs a facilitator discovery to find a device with higher centrality in the neighboring fog and a Pending Interest Table (PIT) entry is created. The process is repeated at each intermediate fog till either the desired content is found or there are no more facilitators to discover within user specified deadline. Once the content is found, it is forwarded to the consumer device following a reverse path using breadcrumbs left in the PIT at each intermediate fog. Similarly, an intermediate fog subsequently populates the corresponding Forwarding Information Base (FIB) entry for the content at its devices.

\section{EXAMPLE: IDENTIFICATION, INCENTIVIZATION, AND COLlaboration SUMmarized}

Figure 4 summarizes altogether the above identification, incentivization and collaboration phases in IS-Fog. The nodes $A-H$ can be considered part of the fog network while $U_{1}-U_{5}$ represent user devices. The nodes $A-D$ form the coalition to cache content which can be typically considered in a smart building scenario with the node $A$ as the high P-CBC node due to its content provision capability. Similarly the nodes $F-H$ are in a coalition of connected vehicles in a typical urban environment with the node $G$ as the high P-CBC gateway to provide content. The node $E$ on the other hand preferred to form a standalone fog with a self-coalition. It does not achieve high utility in case there is already a high utility coalition $(A-D)$ nearby. Thus, it can be incentivized to merge into the high utility coalition to increase its reward as well as the overall amount of cached content locally. Finally, for any user device from $U_{1}-U_{5}$ can retrieve the desired content using 
the "any-cast" approach from the nearby coalition of nodes collaboratively caching the content.

Self stabilization for failure recovery: It is to note that fogs do not totally replace the infrastructure network, though they can complement the existing infrastructure. Highly time sensitive interests are directly sent to infrastructure, of course with a higher content retrieval cost. For example, in case the desired content is not found at a nearby fog within a certain deadline and no more high centrality devices can be discovered, the content is requested from the infrastructure. Similarly, parts of high resource consuming (computing, caching, etc.) tasks unbearable by user devices are offloaded at the infrastructure thereby assisting the distributed fogs.

\section{PERFormance EVAluation}

We evaluated IS-Fog on scalable vehicular data set using realistic mobility trace from Köln, Germany [14]. The simulations are performed using NS-3. The total number of vehicles in our analysis are 2, 986 for one hour simulation duration with one second of time granularity. The city center is simulated by clustering an area of $6 \times 6 \mathrm{~km}^{2}$. The number of neighborhoods can vary between different cities depending on the size, though we divide it into 36 neighborhoods (fogs).

\section{A. Simulation Scenario}

The interests are generated by 900 nodes (approx. $30 \%$ out of the 2,986 nodes) as consumers in an urban environment using the Zipf distribution with skewness parameter $\alpha=1$ for up to $10^{6}$ content chunks each of $1 K b$ size . We allow $900(30 \%)$ of the nodes to perform in-network caching where uniform buffer sizes are assigned to each node and the remaining nodes are intermediate nodes with no caching so that the nodes caching content can be evaluated efficiently. The corresponding coalition utility is derived for spatio-temporally co-located nodes in each fog. We use our social aware routing protocol STRIVE [15] to forward the user interests and retrieve content from caching nodes.

The Fogs formed using IS-Fog are compared with the following schemes:

- Centrality-based Fog: Degree, Closeness, Betweenness, Eigenvector centrality based fogs.

- No Fog: No coalition is formed, i.e. greedy noncollaborative individual node LRU-based caching policy.

\section{B. Simulation Results}

1) Cache Hits: Figure 5a depicts the average cache hit rate in percentage achieved by nodes in different coalitions (fogs) formed at different times, where the cache hits are shown after each 10 each minutes. This analysis clearly shows that the coalitions formed at the nodes using IS-Fog outperforms existing schemes with a minimum of $60 \%$ and up to $85 \%$ cache hits. Moreover, the case of no coalition between nodes resulted in the poorest performance in terms of cache hits reaching less than $10 \%$ during the entire simulation. Existing social metrics such as typical centrality schemes yielded a cache hit rate of around $20-30 \%$, thus, validating IS-Fog efficiency.

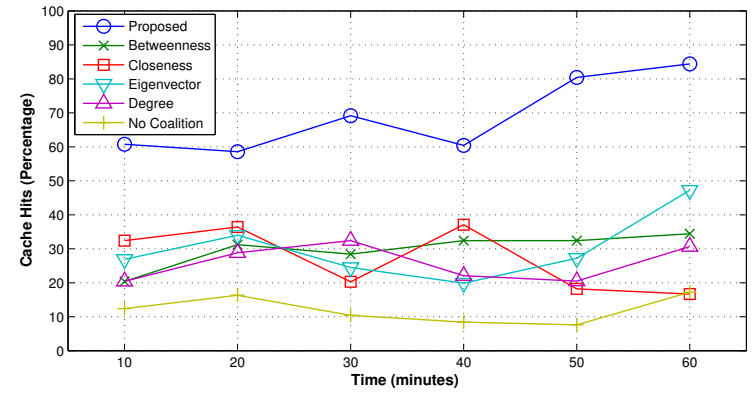

(a) Average cache hit rate

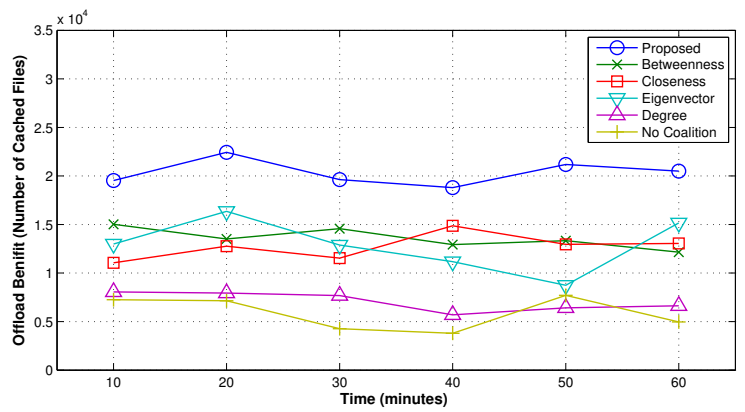

(b) Offload benefit (Content cached locally)

Figure 5: Comparison results of IS-Fog vs No Fog-based caching

2) Offload Benefit: The benefit of offloading at mobile fogs is validated by finding the total number of files cached in the fogs. Figure $5 \mathrm{~b}$ shows the offload benefit achieved by forming different coalitions at each 10 minute interval the simulation. The total number of content files made available for the user by caching at nodes by IS-Fog is higher than with all existing schemes as well as in the case of no coalition.

\section{OPEN RESEARCH ISSUES}

IS-Fog is a caching solution in Information Centric Fog Network. Further research is needed regarding:

1) Resources Management: There is a need to further study how to manage resources, in particular minimizing the computing, caching, communications and energy resource usage of constrained devices in the Internet of Everything (IoE) context. The data centric model of ICN already helps in reducing the excessive resource used, although further measures are needed to enable scalable fog networking solutions.

2) Delay: Delay sensitive applications such as video streaming, Virtual / Augmented Reality (VR/AR), real time multimedia, safety in vehicular, industrial sensors. The basic question still needs an answer of how much delay is a delay for different applications?

3) Limited and intermittent connectivity: This in another key constraint which concerns distributed networking, heterogeneity and mobility experienced by vehicles, mobile phones, etc. There is a need to deal with dynamic environment where the wireless access, devices, and resources in the fog are challenging to manage. 
4) Incentives to cooperate for resource pooling: It is important to provide incentives to different users in order to join fog and provide their resources. The fear of starvation still needs to be addressed as users are reluctant to "give" resources. We already addressed one aspect of incentivizing users with coalition game theory, though there is still the need for the development of efficient metrics with different game-theoretic tools to look after heterogeneity in resources of different users.

5) Spatio-temporal social awareness: There is a need for a user centric approach to study human behavior and social trends in order to be adaptable to user requirements for applications such as crowdsourcing. Also, it is important to focus on both spatial for location awareness and temporal for timeliness aspect of user(s) social interests.

6) Cloud-fog interplay This deals with the interconnections and interactions in fogs. There is a need for efficient content distribution specially Over the top (OTT) content management. It is also important to define when to offload computation and caching from fog to cloud in order to facilitate big data analysis at cloud, Furthermore, there is room to study the orchestration problem in fog network.

7) Security and Privacy: Efficient trust models are needed to cater the existence of malicious nodes along developing solutions to handle byzantine environments in a fog network. Moreover, with the adaption of the General Data Protection Regulation (GDPR) by the European Union, novel data anonymization and encryption mechanism are required to protect user privacy against any breaches in the data collected by various Fog-based applications.

\section{COnclusions}

Despite the evolution towards 5G and beyond, it is challenging for the current infrastructure networks to cope with the bandwidth pressure of the increasing number of connected wireless devices. Existing cloud solutions do not scale well for efficient content provisioning and caching for future AR/VR based multimedia rich applications demanding content "now and here." We introduced IS-Fog as a novel paradigm of leveraging Information Centric Networking to co-exist with fog networking in a social aware approach. The idea is to allow edge devices with sufficient capabilities to collaboratively provide their caching, computing and communications resources for local content provision to nearby users as a fog network. To do this, first we identified such devices using new content-based centrality and model a coalition game to provide them incentives to self-organize and form a fog network to cache content near other user devices. Then, we used both the inter-fog and intra-fog interactions to collaboratively cache and retrieve content from the locally formed fogs. The proposed IS-Fog framework is simulated using large scale realistic traces comprising 2,986 vehicles in an urban environment. Results shown that the proposed framework is an efficient and scalable local content caching and retrieval scheme.

\section{REFERENCES}

[1] E. Baccarelli, P. G. V. Naranjo, M. Scarpiniti, M. Shojafar, and J. H. Abawajy, "Fog of everything: Energy-efficient networked computing architectures, research challenges, and a case study," IEEE Access 5, 9882-9910, 2017.

[2] A. Gharaibeh, M. A. Salahuddin, S. J. Hussini, A. Khreishah, I. Khalil, M. Guizani, and A. Al-Fuqaha, "Smart cities: A survey on data management, security, and enabling technologies," IEEE Communications Surveys \& Tutorials, vol. 19, no. 4, pp. 2456-2501, 2017.

[3] C. white paper, "Fog computing and the internet of things: Extend the cloud to where the things are," Retrieved on June 04, 2019, https://www.cisco.com/c/dam/en_us/solutions/trends/iot/docs/ computing-overview.pdf, 2019.

[4] C. Mouradian, D. Naboulsi, S. Yangui, R. H. Glitho, M. J. Morrow, and P. A. Polakos, "A comprehensive survey on fog computing: Stateof-the-art and research challenges," IEEE Communications Surveys \& Tutorials, 20(1), 416-464, 2017.

[5] I. Abdullahi, S. Arif, and S. Hassan, "Ubiquitous shift with information centric network caching using fog computing," in Computational intelligence in information systems. Springer, Cham, pp. 327-335, 2015.

[6] B. Ahlgren, C. Dannewitz, C. Imbrenda, D. Kutscher, and B. Ohlman, "A survey of information-centric networking," Communications Magazine, IEEE, vol. 50, no. 7, pp. 26-36, 2012.

[7] A. Seetharam, "On caching and routing in information-centric networks,” IEEE Communications Magazine, 56(3), 204-209, 2017.

[8] I. U. Din, S. Hassan, M. K. Khan, M. Guizani, O. Ghazali, and A. Habbal, "Caching in information-centric networking: Strategies, challenges, and future research directions," IEEE Communications Surveys \& Tutorials 20(2), 1443-1474, 2017.

[9] L. Page, S. Brin, R. Motwani, and T. Winograd, "The pagerank citation ranking: Bringing order to the web.” Stanford InfoLab, Tech. Rep., 1999.

[10] L. Lü, D. Chen, X.-L. Ren, Q.-M. Zhang, Y.-C. Zhang, and T. Zhou, "Vital nodes identification in complex networks," Physics Reports, vol. 650, pp. 1-63, 2016.

[11] M. Amadeo, A. Giordano, C. Mastroianni, and A. Molinaro, "On the integration of information centric networking and fog computing for smart home services," in The Internet of Things for Smart Urban Ecosystems. Springer, 2019, pp. 75-93.

[12] J. A. Khan and Y. Ghamri-Doudane, "Saving: socially aware vehicular information-centric networking," IEEE Communications Magazine, vol. 54, no. 8, pp. 100-107, 2016.

[13] J. Li, H. Chen, Y. Chen, Z. Lin, B. Vucetic, and L. Hanzo, "Pricing and resource allocation via game theory for a small-cell video caching system," IEEE Journal on Selected Areas in Communications, vol. 34, no. 8, pp. 2115-2129, 2016.

[14] S. Uppoor, O. Trullols-Cruces, M. Fiore, and J. M. Barcelo-Ordinas, "Generation and analysis of a large-scale urban vehicular mobility dataset," Mobile Computing, IEEE Transactions on, vol. 13, no. 5, pp. 1061-1075, 2014.

[15] J. A. Khan and Y. Ghamri-Doudane, "Strive: Socially-aware threetier routing in information-centric vehicular environment," in Global Communications Conference (GLOBECOM), 2016 IEEE. IEEE, 2016, pp. 1-7.

Junaid Ahmed Khan is a postdoctoral fellow at Center for Urban Science and Progress (CUSP), New York University, NY, USA. His research interests are Information Centric Networks, Connected Autonomous Vehicles (CAVs), Fog Networking, Internet of Things (IoT), and complex/social networks analysis. Cedric Westphal is a Principal Research Architect with Huawei Innovations working on future network architectures, both for wired and wireless networks. His current focus is on Information Centric Networks. He also has been an adjunct assistant professor with the University of California, Santa Cruz since 2009.

Yacine Ghamri-Doudane is currently a full professor at the University of La Rochelle and director of its Laboratory of Informatics, Image and Interaction. His current research interests lie in the area of wireless networking and mobile computing, with a current emphasis on topics related to IoT, wireless sensor networks, and vehicular networks. 